\title{
The neighbourhood effects of geographical access to tobacco retailers on individual smoking behaviour
}

Jamie Pearce, Rosemary Hiscock, Graham Moon and Ross Barnett

J Epidemiol Community Health published online 15 Jul 2008;

doi:10.1136/jech.2007.070656

Updated information and services can be found at:

http://jech.bmj.com/cgi/content/abstract/jech.2007.070656v1

These include:

Rapid responses You can respond to this article at:

http://jech.bmj.com/cgi/eletter-submit/jech.2007.070656v1

Email alerting Receive free email alerts when new articles cite this article - sign up in the box at the service top right corner of the article

Notes

Online First contains unedited articles in manuscript form that have been peer reviewed and accepted for publication but have not yet appeared in the paper journal (edited, typeset versions may be posted when available prior to final publication). Online First articles are citable and establish publication priority; they are indexed by PubMed from initial publication. Citations to Online First articles must include the digital object identifier (DOIs) and date of initial publication.

To order reprints of this article go to:

http://journals.bmj.com/cgi/reprintform

To subscribe to Journal of Epidemiology and Community Health go to:

http://journals.bmj.com/subscriptions/ 


\title{
The neighbourhood effects of geographical access to tobacco retailers on individual smoking behaviour
}

\author{
Jamie Pearce $^{1}$, Rosemary Hiscock ${ }^{1}$, Graham Moon $^{2}$, Ross Barnett ${ }^{1}$ \\ 1. GeoHealth Laboratory, \\ Department of Geography, \\ University of Canterbury, \\ New Zealand. \\ 2. Centre for Geographical Health Research \\ School of Geography, \\ University of Southampton, \\ United Kingdom.
}

The Corresponding Author has the right to grant on behalf of all authors and does grant on behalf of all authors, an exclusive licence (or non exclusive for government employees) on a worldwide basis to the BMJ Publishing Group Ltd and its Licensees to permit this article (if accepted) to be published in JECH editions and any other BMJPGL products to exploit all subsidiary rights, as set out in our licence (http://jech.bmjjournals.com/ifora/licence.pdf)"

Keywords: Neighbourhoods; smoking; deprivation; accessibility; Geographic Information Systems

Correspondence to: Jamie Pearce, GeoHealth Laboratory, Department of Geography, University of Canterbury, Private Bag 4800, Christchurch 8020, New Zealand. jamie.pearce@ canterbury.ac.nz Tel: +64 33642987 ext:7943

Fax: +64 33642907 


\begin{abstract}
Objective: To investigate whether neighbourhood measures of geographical accessibility to outlets selling tobacco (supermarkets, convenience stores and petrol stations) are associated with individual smoking behaviour in New Zealand.

Methods: Using Geographical Information Systems, travel times from the population-weighted centroid of each neighbourhood to the closest outlet selling tobacco were calculated for all 38,350 neighbourhoods across New Zealand. These measures were appended to the 2002/03 New Zealand Health Survey; a national survey of 12,529 adults. Two-level logistic regression models were fitted to examine the effects of neighbourhood locational access upon individual smoking behaviour after controlling for potential individual- and neighbourhood-level confounding factors, including deprivation and urban/rural status.

Results: After controlling for individual-level demographic and socioeconomic variables, individuals living in the quartiles of neighbourhoods with the best access to supermarkets (OR 1.23, 95\% CI 1.06-1.42) and convenience stores (OR 1.19, 95\% CI 1.03-1.38) had a higher odds of smoking compared to individuals in the worst access quartiles. However, the association between neighbourhood accessibility to supermarkets and convenience stores was not apparent once other neighbourhood-level variables (deprivation and rurality) were included.

Conclusions: At the national level, there is little evidence to suggest that after adjustment for neighbourhood deprivation better locational access to tobacco retail provision in New Zealand is associated with individual-level smoking behaviour.
\end{abstract}




\section{Introduction}

Smoking is one of the most important public health issues worldwide and is a major determinant of preventable mortality and morbidity in developed and developing countries.[1] Disparities in tobacco consumption between different social and ethnic groups have been noted in a number of countries.[2] Because the environments in which people live influence many health behaviours, [3] there has been an increasing focus on how neighbourhood characteristics may shape the consumption of tobacco, and, by extension, how neighbourhood change might reduce the burden of smoking-related health outcomes and geographical inequalities in health. Recent studies have identified a variety of mechanisms that operate through residential neighbourhoods to effect smoking behaviour after controlling for individual characteristics. Neighbourhood characteristics including area disadvantage,[4-12] social and economic inequality,[13-15] residential segregation,[16] physical stressors such as crime,[10, 17, 18] and urban/rural status,[19-21] have been found to influence individual smoking behaviour.

Although there has been considerable interest in 'place effects' on individual smoking behaviour, most previous studies have relied upon characteristics of neighbourhoods that are provided in routinely collected data sources, particularly the census. This approach can be criticised for failing to identify and measure other important features of neighbourhoods that may partially contribute to the smoking behaviour of local residents. This limitation may be a significant obstacle to successful place-based policy initiatives.

One area characteristic that has received limited attention is the influence of neighbourhood availability of outlets selling tobacco. We hypothesise that retail availability, other things being equal, could increases the likelihood of consumption in a number of ways. First, an effect of neighbourhood access on smoking rates is particularly likely given the international evidence which indicates that outlets selling tobacco are often disproportionately located in more deprived communities.[22] This observation is true in New Zealand where supermarkets and convenience stores (the major retailers of tobacco) are more accessible [23] and more concentrated[24] in socially deprived neighbourhoods. Increased opportunities to procure tobacco thus may affect consumption levels through providing an environment that supports easy access to tobacco products. Research in the alcohol literature suggests that physical neighbourhood availability of alcohol products affects individual consumption and related behaviours, $[25,26]$ and this could also be true of smoking. The effects of increased access should be particularly important among younger smokers or sole parents in deprived neighbourhoods who are more likely to lack available transport and rely on readily accessible local supplies. Secondly, smokers are price responsive and low income communities are particularly price sensitive,[27] so a more competitive local market may help stimulate increased levels of consumption. Third, the greater concentration of outlets in deprived neighbourhoods may accentuate the scope for addiction, making it harder for people to quit especially when cigarettes are available in the local facilities which they are more likely utilise.

Only one previous study has examined the effect of neighbourhood access to outlets selling tobacco on individual smoking behaviour. In this study of 82 neighbourhoods in four northern Californian cities, neighbourhood measures of convenience store access (distance to and concentration of) were significantly associated with higher levels of individual-level smoking after controlling for individual characteristics.[11] The association between convenience store 
access and individual smoking was modified by individual and neighbourhood socioeconomic status (SES) as the effects of store access were, unexpectedly, only apparent in high SES neighbourhoods. The study considered only one type of tobacco retailer (convenience stores), did not include supermarkets, drug stores and liquor stores, and was limited to a specific subnational context.

The limited attention to the effects of locational availability of tobacco on individual smoking behaviour is surprising given the considerable attention provided to examining the influence of geographical access to other neighbourhood resources such as green space, food stores and health service provision on various individual health outcomes. For example, there is evidence that the nature of the 'foodscape' influences individual-level nutrition- and obesity-related health outcomes in some,[28-30] but not all,[31,32] settings.

This paper addresses this knowledge gap for tobacco consumption through the provision of a national level study. Our focus is New Zealand where smoking is responsible for approximately $15 \%$ of all deaths,[33] and there is a strong and rising social gradient in tobacco consumption, with higher rates among lower socioeconomic groups,[34] among Māori,[35] and for those living in more socially deprived neighbourhoods.[36] A study of two large national cohorts found that the contribution of tobacco smoking to inequalities in mortality increased between 1984 and 1999 from 16 to 21 percent for males and 3 to 11 percent for females.[34] Prevalence rates are approximately twice as high in the most deprived quintile of neighbourhoods compared to the least deprived,[37] probably contributing to increasing geographical inequalities in health in New Zealand.[38, 39] A high priority is, for these reasons, placed on reducing the prevalence of smoking.[40] We examine whether access to outlets selling tobacco in neighbourhoods across New Zealand is associated with individual-level smoking behaviour after adjustment for potential individual- and neighbourhood-level confounders.

\section{Methods}

Data on the addresses of each supermarket and local convenience store (including small corner stores, petrol stations and mini-markets) were collected during 2004 from all 74 Territorial Authorities (TAs) across New Zealand. TAs have regulatory responsibility for the hygiene inspection of all premises in their region used in the manufacture, preparation or storage of food for sale. To allow the food outlet data to be geocoded, information was requested on the street address. There were a total of 661 supermarkets and 3681 convenience stores. Geographical access to supermarkets and convenience stores was calculated separately for all 38,350 census meshblocks (average population 100), or what we refer to as 'neighbourhoods'. Each neighbourhood was represented by its population-weighted centroid and the travel time (by car) taken to the nearest supermarket and convenience store along the road network was calculated using the network functionality in a Geographical Information System (GIS). All segments in the road system were adjusted to account for variations in speed limits, type of road surface, sinuosity and differences in the topography across the network.[41] 
The 2002/03 New Zealand Health Survey (NZHS) is a national survey of the health status of 12,529 adults aged 15 years and over living in non-institutional permanent dwellings (target population 2.6 million) posing a range of questions that include smoking behaviour.[37] The 12,529 respondents were distributed across 1,178 meshblock neighbourhoods and there were between 1 and 83 respondents per neighbourhood, although in most neighbourhoods there were less than 20 respondents. Each individual in the survey was defined as a 'smoker' or 'non smoker' depending on their response to the question 'Do you smoke one or more tobacco cigarettes a day?'. Each smoker was categorised as a 'light' or 'heavy' (>10 cigarettes per day) smoker. The neighbourhood measures of tobacco outlet access were divided into quartiles (for confidentiality reasons) and appended to each respondent in the survey based on the neighbourhood that they lived in. The individual-level variables from the Health Survey and the neighbourhood-level measures that were examined in this study are summarised in Table 1 (the NZHS deliberately over-sampled ethnic minority populations and furthermore has a complex sampling frame. Therefore, the figures in the table relate only to the survey and cannot be extrapolated to the New Zealand population).

\section{Analyses}

Two-level logistic regression models with a random intercept were fitted in MLWin using second order penalised quasilikelihood (PQL) estimation methods. Our two dependent variables were examined separately in view of the different processes underpinning the decision to smoke, and the extent of smoking. First, we considered whether there were neighbourhood effects on all 'smokers' compared to 'non-smokers'. Second, we examined the effects on 'heavy smokers' versus all other respondents (i.e. light and non-smokers).

Variables were added in four stages. First, we included, sex, age, and design variables (ethnic composition of the neighbourhood, number of respondents from the neighbourhood, number of adults in the household, and respondent prioritised ethnicity) to account for the sample stratification and oversampling of ethnic minorities. Second, individual-level socioeconomic variables were added: education (none, school, post-school), social class (professional/managerial, other non-manual, skilled manual, semi and unskilled manual, and unavailable), receipt of poverty-related benefits (family support, domestic purposes or unemployment) in the last year (recipient or not), and household income $(<\$ 25 \mathrm{k}, \$ 25-50 \mathrm{k}$, $>\$ 50 \mathrm{k}$ ). Two potential ecological confounders (at the neighbourhood-level) were added in the third and fourth stages respectively: area deprivation measured using the $2001 \mathrm{New}$ Zealand Deprivation Index (NZDep)[42] divided into quintiles, and the 2001 Urban-Rural Area Classification, (main urban area, secondary urban area, minor urban area, rural centre, highly rural).[43] Due to multicollinearity we could not include both supermarkets and convenience stores in the same model; therefore, results are reported separately. There was also a substantive justification for this decision in that tobacco purchases at supermarkets are generally the result of a multipurpose shopping trip where many other products may be bought. Trips to convenience stores are usually for one or two purchases. This distinction indicates that convenience store purchases of tobacco might be more likely to be subject to access constraints. In order to examine whether SES moderated the relationship between locational access and 
individual smoking behaviour, potential interactions between the main effects (access to supermarkets and convenience stores) and the socioeconomic variables were also examined.

Table 1. Summary information for individuals in the 2002-03 New Zealand Health Survey $(n=12,529)$.

\begin{tabular}{|c|c|c|c|c|c|}
\hline Variable & Count & $\%$ & & Count & $\%$ \\
\hline Sex & & & \multicolumn{3}{|l|}{ Benefits } \\
\hline Female & 7658 & 61.1 & Non-recipient & 9784 & 78.1 \\
\hline Male & 4871 & 38.9 & \multirow[t]{2}{*}{ Recipient } & 2745 & 21.9 \\
\hline Total & 12529 & 100.0 & & & \\
\hline Age group & & & \multicolumn{3}{|l|}{ Household income $(N Z \$)$} \\
\hline $65+$ & 2206 & 17.6 & \multirow{4}{*}{$\begin{array}{l}>50,000 \\
25,000-50,000 \\
<25,000\end{array}$} & 3948 & 31.5 \\
\hline $45-64$ & 3718 & 29.7 & & 3842 & 30.7 \\
\hline $25-44$ & 5039 & 40.2 & & 4730 & 37.8 \\
\hline $15-24$ & 1566 & 12.5 & & & \\
\hline Ethnicity & & & \multicolumn{3}{|l|}{ Education } \\
\hline Other & 6329 & 50.5 & \multirow{4}{*}{$\begin{array}{l}\text { Tertiary qualifications } \\
\text { School qualifications only } \\
\text { No qualifications }\end{array}$} & 5581 & 44.6 \\
\hline Māori & 4120 & 32.9 & & 3257 & 26.0 \\
\hline Pacific & 908 & 7.2 & & 3681 & 29.4 \\
\hline Asian & 1172 & 9.4 & & & \\
\hline Social Class & & & \multicolumn{3}{|l|}{ Current smoker } \\
\hline Managerial & 2594 & 20.7 & Yes & 3739 & 29.9 \\
\hline Other non-manual & 3084 & 24.6 & No & 8785 & 70.1 \\
\hline Skilled manual & 1699 & 13.6 & \multicolumn{3}{|c|}{ Heavy smoker (>10 cigarettes per day) } \\
\hline Semi \& unskilled manual & 1958 & 15.6 & Yes & 1789 & 14.3 \\
\hline Unavailable & 3194 & 25.5 & No & 10719 & 85.7 \\
\hline \multicolumn{6}{|l|}{ Neighbourhood-level variables } \\
\hline Neighbourhood Deprivation & & & \multicolumn{3}{|c|}{ Neighbourhood Access to Supermarkets(mins) } \\
\hline 1 (least deprived) & 1705 & 13.6 & Worst 6.54 & 2733 & 21.9 \\
\hline 2 & 1573 & 12.6 & Worse 3.22-6.54 & 3387 & 27.1 \\
\hline 3 & 1854 & 14.8 & Better 1.89-3.22 & 3444 & 27.6 \\
\hline 4 & 2389 & 19.1 & Best $<1.89$ & 2933 & 23.5 \\
\hline 5 (most deprived) & 4979 & 39.8 & & & \\
\hline Neighbourhood Urban/Rural Status & & & \multicolumn{3}{|c|}{ Neighbourhood Access to Convenience Stores (mins) } \\
\hline Main urban & 7650 & 61.1 & Worst 3.89+ & 2409 & 19.3 \\
\hline Secondary urban & 724 & 5.8 & Worse 1.65-3.89 & 3173 & 25.4 \\
\hline Minor urban & 1501 & 12.0 & Better 0.98-1.65 & 3520 & 28.2 \\
\hline Rural centre & 706 & 5.6 & Best $<0.98$ & 3371 & 27.0 \\
\hline Highly rural & 1948 & 15.5 & & & \\
\hline
\end{tabular}

The figures in the table relate only to the survey and cannot be extrapolated to the New Zealand population

Odds ratios and $95 \%$ confidence intervals were calculated for quartile measures of access to supermarkets and access to convenience stores. The three quartiles with the best access to a supermarket or convenience store were compared to the worst access quartile (greatest travel time). 


\section{Results}

\section{Supermarkets}

We found that in the baseline model (that controlled for the design variables, age and sex) smoking was moderately associated with neighbourhood access to supermarkets (Model 1, Table 2). Compared to the reference category (worst access), the odds ratios for each quartile increased with better levels of access in an approximately linear fashion. However, the confidence intervals suggest that access only affects smoking for respondents in the two most accessible quartiles of neighbourhoods. The odds ratio in the quartile of neighbourhoods with the best access to a supermarket compared to the base category suggests that residents of these neighbourhoods had a 22 percent higher odds of being a smoker. The modest association between supermarket accessibility and smoking remained once individual-level socioeconomic status was controlled for (Model 2). However, any gradient relationship between access and smoking weakened on the addition of neighbourhood deprivation (Model 3) and disappeared when rurality was included (Model 4). At the same time controlling for area deprivation (Model 3 ) and area type (Model 4) removed any suggestion of statistical significance in the relationship between smoking and supermarket access.

Similar findings were noted for heavy smokers compared to light and non-smokers (Table 3). In the baseline model the odds ratio of being a heavy smoker was higher in neighbourhoods with better access to supermarkets (Model 1). Relative to the base category (worst access), the odds ratio increased from 1.05 in quartile 2 to 1.28 in quartile 4 (neighbourhoods with the best access), although the confidence intervals for all quartiles (except for the most accessible quartile of neighbourhoods) included 1.0. This pattern of relationship persisted in Models 2 and 3; in Model 4 even the confidence interval for the most accessible quartile overlapped 1.0, although the odds ratios for the accessibility quartiles continued to exhibit a small gradient ranging from 1.04 in quartile 2 to 1.15 in quartile 4.

To probe this finding further, we explored additional models (not shown). Both outcomes were modelled with access omitted. No significant change was observed for the individual or neighbourhood socio-economic deprivation coefficients, confirming that access represents a minor, non-significant attenuation or compounding of stronger relationships with socioeconomic deprivation, mainly at an area level. Second, we considered possible interactions between access to supermarkets and individual- or area-level socioeconomic variables. There was some suggestion in the heavy smoking model of significant interaction between supermarket access and individual educational qualification and between supermarket access and neighbourhood deprivation. In both cases, however, confidence intervals overlapped extensively and there was no coherent patterning to the results. Finally, we examined the relationship between neighbourhood access to supermarkets and smoking status/heavy smoking for urban areas only. The results were consistent with the national findings. 
Table 2. Odds ratio of being a smoker (95\% confidence intervals) and random variance estimates predicted from access to supermarkets in the 2002-03 New Zealand Health Survey $(\mathrm{n}=12,529)$.

\begin{tabular}{|c|c|c|c|c|}
\hline & $\begin{array}{l}\text { Model 1 }{ }^{\mathrm{a}} \\
\text { Baseline }\end{array}$ & $\begin{array}{l}\text { Model } 2^{\mathrm{b}} \\
\text { Individual socio- } \\
\text { economic status }\end{array}$ & $\begin{array}{c}\text { Model } 3^{\mathrm{c}} \\
\text { Area deprivation }\end{array}$ & $\begin{array}{l}\text { Model } 4^{\mathrm{d}} \\
\text { Urban/rural }\end{array}$ \\
\hline \multicolumn{5}{|l|}{ Neighbourhood Access (mins) } \\
\hline Worst $>6.54$ & 1 & 1 & 1 & 1 \\
\hline Worse $3.22-6.54$ & $1.08(0.93-1.25)$ & $1.12(0.97-1.29)$ & $1.08(0.94-1.24)$ & $1.03(0.86-1.24)$ \\
\hline Better 1.89-3.22 & $1.17(1.01-1.35)$ & $1.19(1.04-1.38)$ & $1.12(0.97-1.29)$ & $1.07(0.89-1.29)$ \\
\hline Best $<1.89$ & $1.22(1.05-1.42)$ & $1.23(1.06-1.42)$ & $1.12(0.97-1.29)$ & $1.06(0.88-1.28)$ \\
\hline \multicolumn{5}{|l|}{$\operatorname{Sex}$} \\
\hline Male & 1 & 1 & 1 & 1 \\
\hline Female & $1.04(0.95-1.14)$ & $1.10(1.00-1.21)$ & $1.10(1.00-1.22)$ & $1.11(1.01-1.22)$ \\
\hline \multicolumn{5}{|l|}{ Age group } \\
\hline $65+$ & 1 & 1 & 1 & 1 \\
\hline $45-64$ & $4.31(3.57-5.21)$ & $3.77(3.06-4.64)$ & $3.68(2.99-4.52)$ & $3.67(2.99-4.52)$ \\
\hline $25-44$ & $4.57(3.91-5.33)$ & $4.38(3.64-5.27)$ & $4.26(3.54-5.13)$ & $4.26(3.54-5.13)$ \\
\hline $15-24$ & $2.86(2.44-3.36)$ & $2.85(2.38-3.40)$ & $2.78(2.33-3.31)$ & $2.78(2.33-3.32)$ \\
\hline \multicolumn{5}{|l|}{ Ethnicity } \\
\hline Other & 1 & 1 & 1 & 1 \\
\hline Māori & $2.94(2.62-3.29)$ & $2.44(2.17-2.74)$ & $2.22(1.97-2.50)$ & $2.22(1.98-2.50)$ \\
\hline Pacific & $1.52(1.27-1.82)$ & $1.28(1.06-1.53)$ & $1.12(0.93-1.35)$ & $1.13(0.94-1.36)$ \\
\hline Asian & $0.37(0.29-0.46)$ & $0.38(0.31-0.48)$ & $0.37(0.30-0.46)$ & $0.38(0.30-0.47)$ \\
\hline \multicolumn{5}{|l|}{ Education } \\
\hline Tertiary qualifications & & 1 & 1 & 1 \\
\hline School qualifications only & & $0.98(0.87-1.09)$ & $0.98(0.87-1.09)$ & $0.98(0.87-1.09)$ \\
\hline No qualifications & & $1.51(1.35-1.69)$ & $1.49(1.34-1.67)$ & $1.49(1.33-1.67)$ \\
\hline \multicolumn{5}{|l|}{ Social Class } \\
\hline Managerial/Professional & & 1 & 1 & 1 \\
\hline Other non-manual & & $1.26(1.10-1.45)$ & $1.24(1.08-1.42)$ & $1.24(1.08-1.42)$ \\
\hline Skilled manual & & $1.81(1.55-2.13)$ & $1.77(1.51-2.08)$ & $1.78(1.52-2.09)$ \\
\hline Semi \& unskilled manual & & $1.71(1.46-2.00)$ & $1.64(1.41-1.92)$ & $1.64(1.40-1.91)$ \\
\hline Unavailable & & $1.11(0.94-1.31)$ & $1.07(0.91-1.26)$ & $1.07(0.91-1.26)$ \\
\hline \multicolumn{5}{|l|}{ Benefits } \\
\hline Non-recipient & & 1 & 1 & 1 \\
\hline Recipient & & $1.53(1.37-1.70)$ & $1.51(1.36-1.68)$ & $1.51(1.36-1.68)$ \\
\hline \multicolumn{5}{|l|}{ Household income } \\
\hline$>50,000$ & & 1 & 1 & 1 \\
\hline $25,000-50,000$ & & $1.20(1.07-1.35)$ & $1.13(1.00-1.27)$ & $1.12(1.00-1.26)$ \\
\hline$<25,000$ & & $1.48(1.30-1.68)$ & $1.36(1.19-1.55)$ & $1.36(1.19-1.55)$ \\
\hline \multicolumn{5}{|l|}{ Neighbourhood Deprivation } \\
\hline 1 (least deprived) & & & 1 & 1 \\
\hline 2 & & & $1.15(0.94-1.41)$ & $1.15(0.94-1.42)$ \\
\hline 3 & & & $1.22(1.00-1.49)$ & $1.22(1.00-1.49)$ \\
\hline 4 & & & $1.64(1.36-1.98)$ & $1.65(1.36-1.99)$ \\
\hline 5 (most deprived) & & & $1.87(1.53-2.29)$ & $1.86(1.52-2.28)$ \\
\hline \multicolumn{5}{|c|}{ Neighbourhood Urban/Rural Status } \\
\hline
\end{tabular}


Secondary urban

$1.12(0.91-1.37)$

Minor urban

$1.02(0.88-1.19)$

Rural centre

$1.08(0.85-1.35)$

Highly rural

Level 2 variance (Std err) $^{\text {e }}$

$0.13(0.03)$

$0.07(0.02)$

$0.05(0.02)$

$0.92(0.76-1.11)$

a. Models include design (to account for sample stratification and oversampling of ethnic minorities), and individual-level sex and age variables.

b. Individual-level socio-economic variables included in models containing design, sex and age variables.

c. Neighbourhood-level deprivation included in models containing individual-level design, sex, age and socioeconomic variables.

d. Neighbourhood-level urban area classification included in models containing neighbourhood-level deprivation and individual-level design, sex, age and socio-economic variables.

e. Variance at the neighbourhood-level 
Table 3. Odds ratio of being a heavy smoker (95\% confidence intervals) and random variance estimates predicted from access to supermarkets in the 2002-03 New Zealand Health Survey $(\mathrm{n}=12,529)$.

\begin{tabular}{|c|c|c|c|c|}
\hline & $\begin{array}{l}\text { Model 1 }{ }^{\mathrm{a}} \\
\text { Baseline }\end{array}$ & $\begin{array}{c}\text { Model } 2^{\mathrm{b}} \\
\text { Individual socio- } \\
\text { economic status }\end{array}$ & $\begin{array}{c}\text { Model } 3^{\mathrm{c}} \\
\text { Area deprivation }\end{array}$ & $\begin{array}{l}\text { Model } 4^{\mathrm{d}} \\
\text { Urban/rural }\end{array}$ \\
\hline \multicolumn{5}{|l|}{ Neighbourhood Access (mins) } \\
\hline Worst $>6.54$ & 1 & 1 & 1 & 1 \\
\hline Worse 3.22-6.54 & $1.05(0.88-1.25)$ & $1.12(0.94-1.33)$ & $1.08(0.91-1.27)$ & $1.04(0.84-1.30)$ \\
\hline Better 1.89-3.22 & $1.16(0.97-1.38)$ & $1.22(1.03-1.44)$ & $1.14(0.96-1.35)$ & $1.10(0.88-1.38)$ \\
\hline Best $<1.89$ & $1.28(1.07-1.52)$ & $1.33(1.12-1.58)$ & $1.20(1.01-1.43)$ & $1.15(0.92-1.44)$ \\
\hline \multicolumn{5}{|l|}{$\operatorname{Sex}$} \\
\hline Male & 1 & 1 & 1 & 1 \\
\hline Female & $0.79(0.71-0.88)$ & $0.87(0.77-0.98)$ & $0.87(0.78-0.98)$ & $0.87(0.78-0.98)$ \\
\hline \multicolumn{5}{|l|}{ Age group } \\
\hline $65+$ & 1 & 1 & 1 & 1 \\
\hline $45-64$ & $3.60(2.88-4.50)$ & $4.01(3.15-5.11)$ & $3.89(3.06-4.96)$ & $3.90(3.07-4.97)$ \\
\hline $25-44$ & $4.57(3.68-5.69)$ & $5.06(3.93-6.50)$ & $4.90(3.81-6.29)$ & $4.91(3.82-6.31)$ \\
\hline $15-24$ & $2.70(2.06-3.53)$ & $2.64(1.98-3.53)$ & $2.56(1.92-3.42)$ & $2.57(1.93-3.43)$ \\
\hline \multicolumn{5}{|l|}{ Ethnicity } \\
\hline Other & 1 & 1 & 1 & 1 \\
\hline Māori & $1.93(1.68-2.23)$ & $1.60(1.38-1.84)$ & $1.43(1.24-1.65)$ & $1.43(1.24-1.65)$ \\
\hline Pacific & $0.83(0.64-1.07)$ & $0.68(0.53-0.88)$ & $0.58(0.45-0.75)$ & $0.59(0.45-0.77)$ \\
\hline Asian & $0.17(0.11-0.25)$ & $0.17(0.11-0.26)$ & $0.16(0.11-0.25)$ & $0.17(0.11-0.25)$ \\
\hline \multicolumn{5}{|l|}{ Education } \\
\hline Tertiary qualifications & & 1 & 1 & 1 \\
\hline School qualifications only & & $0.99(0.85-1.14)$ & $0.99(0.85-1.14)$ & $0.99(0.86-1.14)$ \\
\hline No qualifications & & $1.48(1.29-1.69)$ & $1.46(1.28-1.67)$ & $1.46(1.27-1.67)$ \\
\hline \multicolumn{5}{|l|}{ Social Class } \\
\hline Managerial/Professional & & 1 & 1 & 1 \\
\hline Other non-manual & & $1.31(1.09-1.57)$ & $1.28(1.06-1.54)$ & $1.28(1.06-1.53)$ \\
\hline Skilled manual & & $2.16(1.77-2.63)$ & $2.10(1.72-2.56)$ & $2.09(1.71-2.55)$ \\
\hline Semi \& unskilled manual & & $1.80(1.48-2.19)$ & $1.71(1.41-2.08)$ & $1.70(1.40-2.07)$ \\
\hline Unavailable & & $1.37(1.11-1.70)$ & $1.31(1.06-1.63)$ & $1.31(1.06-1.62)$ \\
\hline \multicolumn{5}{|l|}{ Benefits } \\
\hline Non-recipient & & 1 & 1 & 1 \\
\hline Recipient & & $1.26(1.11-1.44)$ & $1.25(1.09-1.42)$ & $1.25(1.09-1.42)$ \\
\hline \multicolumn{5}{|l|}{ Household income } \\
\hline$>50,000$ & & 1 & 1 & 1 \\
\hline $25,000-50,000$ & & $1.30(1.12-1.51)$ & $1.21(1.04-1.40)$ & $1.20(1.04-1.40)$ \\
\hline$<25,000$ & & $1.50(1.27-1.76)$ & $1.36(1.15-1.61)$ & $1.35(1.15-1.60)$ \\
\hline \multicolumn{5}{|l|}{ Neighbourhood Deprivation } \\
\hline 1 (least deprived) & & & 1 & 1 \\
\hline 2 & & & $1.22(0.93-1.60)$ & $1.22(0.93-1.60)$ \\
\hline 3 & & & $1.17(0.90-1.52)$ & $1.18(0.90-1.53)$ \\
\hline 4 & & & $1.72(1.34-2.20)$ & $1.72(1.35-2.20)$ \\
\hline 5 (most deprived) & & & $2.06(1.59-2.68)$ & $2.06(1.59-2.67)$ \\
\hline \multicolumn{5}{|c|}{ Neighbourhood Urban/Rural Status } \\
\hline
\end{tabular}


Secondary urban

$1.24(0.98-1.57)$

Minor urban $1.09(0.92-1.30)$

Rural centre $1.06(0.81-1.38)$

Highly rural

Level 2 variance $^{e}$ (Std err)

$0.13(0.04)$

$0.07(0.03)$

$0.05(0.03)$

$0.98(0.78-1.24)$

a. Models include design (to account for sample stratification and oversampling of ethnic minorities), and individual-level sex and age variables.

b. Individual-level socio-economic variables included in models containing design, sex and age variables.

c. Neighbourhood-level deprivation included in models containing individual-level design, sex, age and socio-economic variables.

d. Neighbourhood-level urban area classification included in models containing neighbourhood-level deprivation and individual-level design, sex, age and socio-economic variables.

e. Variance at the neighbourhood-level 
Table 4. Odds ratio of being a smoker (95\% confidence intervals) and random variance estimates predicted from access to convenience stores in the 2002-03 New Zealand Health Survey $(\mathrm{n}=12,529)$.

\begin{tabular}{|c|c|c|c|c|}
\hline & $\begin{array}{l}\text { Model } 1^{\mathrm{a}} \\
\text { Baseline }\end{array}$ & $\begin{array}{c}\text { Model } 2^{\mathrm{b}} \\
\text { Individual socio- } \\
\text { economic status }\end{array}$ & $\begin{array}{c}{\text { Model } 3^{\mathrm{c}}} \\
\text { Area deprivation }\end{array}$ & $\begin{array}{c}\text { Model } 4^{\mathrm{d}} \\
\text { Urban/rural }\end{array}$ \\
\hline \multicolumn{5}{|l|}{ Neighbourhood Access (mins) } \\
\hline Worst $>3.89$ & 1 & 1 & 1 & 1 \\
\hline Worse $1.65-3.89$ & $1.10(0.95-1.28)$ & $1.12(0.97-1.29)$ & $1.07(0.93-1.23)$ & $0.97(0.82-1.15)$ \\
\hline Better 0.98-1.65 & $1.13(0.97-1.32)$ & $1.15(0.99-1.33)$ & $1.05(0.91-1.22)$ & $0.95(0.79-1.13)$ \\
\hline Best $<0.98$ & $1.18(1.01-1.37)$ & $1.19(1.03-1.38)$ & $1.05(0.91-1.22)$ & $0.95(0.80-1.13)$ \\
\hline \multicolumn{5}{|l|}{ Sex } \\
\hline Male & 1 & 1 & 1 & 1 \\
\hline Female & $1.04(0.96-1.14)$ & $1.11(1.00-1.22)$ & $1.11(1.01-1.22)$ & $1.11(1.01-1.22)$ \\
\hline \multicolumn{5}{|l|}{ Age group } \\
\hline $65+$ & 1 & 1 & 1 & 1 \\
\hline $45-64$ & $2.86(2.44-3.35)$ & $2.84(2.38-3.39)$ & $2.77(2.32-3.31)$ & $2.78(2.33-3.32)$ \\
\hline $25-44$ & $4.53(3.88-5.29)$ & $4.35(3.61-5.23)$ & $4.24(3.53-5.10)$ & $4.25(3.53-5.11)$ \\
\hline $15-24$ & $4.27(3.53-5.15)$ & $3.74(3.03-4.60)$ & $3.66(2.97-4.50)$ & $3.65(2.97-4.50)$ \\
\hline \multicolumn{5}{|l|}{ Ethnicity } \\
\hline Other & 1 & 1 & 1 & 1 \\
\hline Māori & $2.93(2.61-3.28)$ & $2.43(2.16-2.73)$ & $2.21(1.96-2.49)$ & $2.22(1.97-2.50)$ \\
\hline Pacific & $1.52(1.27-1.82)$ & $1.28(1.07-1.54)$ & $1.12(0.93-1.35)$ & $1.13(0.94-1.36)$ \\
\hline Asian & $0.37(0.29-0.46)$ & $0.38(0.31-0.48)$ & $0.37(0.30-0.47)$ & $0.38(0.30-0.47)$ \\
\hline \multicolumn{5}{|l|}{ Education } \\
\hline Tertiary qualifications & & 1 & 1 & 1 \\
\hline School qualifications only & & $0.97(0.87-1.09)$ & $0.97(0.87-1.09)$ & $0.97(0.87-1.09)$ \\
\hline No qualifications & & $1.49(1.33-1.67)$ & $1.48(1.32-1.65)$ & $1.48(1.32-1.66)$ \\
\hline \multicolumn{5}{|l|}{ Social Class } \\
\hline Managerial/Professional & & 1 & 1 & 1 \\
\hline Other non-manual & & $1.27(1.10-1.45)$ & $1.24(1.08-1.43)$ & $1.24(1.08-1.43)$ \\
\hline Skilled manual & & $1.83(1.56-2.15)$ & $1.78(1.52-2.09)$ & $1.79(1.53-2.11)$ \\
\hline Semi \& unskilled manual & & $1.73(1.48-2.02)$ & $1.66(1.42-1.94)$ & $1.65(1.42-1.93)$ \\
\hline Unavailable & & $1.12(0.95-1.32)$ & $1.08(0.92-1.28)$ & $1.08(0.91-1.28)$ \\
\hline \multicolumn{5}{|l|}{ Benefits } \\
\hline Non-recipient & & 1 & 1 & 1 \\
\hline Recipient & & $1.52(1.37-1.70)$ & $1.50(1.35-1.68)$ & $1.50(1.35-1.68)$ \\
\hline \multicolumn{5}{|l|}{ Household income } \\
\hline$>50,000$ & & 1 & 1 & 1 \\
\hline $25,000-50,000$ & & $1.20(1.07-1.35)$ & $1.13(1.00-1.27)$ & $1.12(1.00-1.26)$ \\
\hline$<25,000$ & & $1.48(1.30-1.68)$ & $1.36(1.19-1.55)$ & $1.36(1.19-1.55)$ \\
\hline \multicolumn{5}{|l|}{ Neighbourhood Deprivation } \\
\hline 1 (least deprived) & & & 1 & 1 \\
\hline 2 & & & $1.15(0.94-1.41)$ & $1.16(0.94-1.42)$ \\
\hline 3 & & & $1.24(1.02-1.52)$ & $1.25(1.02-1.53)$ \\
\hline 4 & & & $1.67(1.39-2.02)$ & $1.69(1.40-2.04)$ \\
\hline 5 (most deprived) & & & $1.91(1.56-2.35)$ & $1.91(1.56-2.35)$ \\
\hline \multicolumn{5}{|c|}{ Neighbourhood Urban/Rural Status } \\
\hline
\end{tabular}


Secondary urban

$1.10(0.90-1.34)$

Minor urban

$1.01(0.87-1.18)$

Rural centre

$1.04(0.84-1.28)$

Highly rural

Level 2 variance (Std err) $^{e}$

$0.14(0.03)$

$0.08(0.02)$

$0.85(0.71-1.02)$

a. Models include design (to account for sample stratification and oversampling of ethnic minorities), and individual-level sex and age variables.

b. Individual-level socio-economic variables included in models containing design, sex and age variables.

c. Neighbourhood-level deprivation included in models containing individual-level design, sex, age and socio-economic variables.

d. Neighbourhood-level urban area classification included in models containing neighbourhood-level deprivation and individual-level design, sex, age and socio-economic variables.

e. Variance at the neighbourhood-level

\section{Convenience stores}

With regards to access to convenience stores, in the baseline model there was a significant association only between individual smoking behaviour and neighbourhoods with the best accessibility (Model 1, Table 4). The odds ratio in the most accessible quartile of neighbourhoods compared to the reference category (worst access) was 1.18 (95\% CI 1.011.37). This association remained once individual socioeconomic status was controlled for (Model 2). However, the inclusion of neighbourhood variables (deprivation and rurality) attenuated the relationship and the 95\% confidence intervals included 1.0 (Models 3 and 4).

There was little evidence of an association between access to convenience stores and individual-level heavy smoking (Table 5). In the baseline model (Model 1), although the quartile of neighbourhoods with better access to outlets selling tobacco each had odds ratios over 1.0, there was not a convincing dose-response relationship. Further, all of the 95\% confidence intervals included the null. Once individual-level socioeconomic status as well as neighbourhood-level deprivation and urban/rural status were included in the model the odds ratios were all close to 1.0 (Model 4).

These findings were probed using the same approach as that used in the analysis of supermarket access. Results were similar suggesting that more complex modelling does not reinstate significant evidence for store access having any independent effect on smoking behaviour. 
Table 5. Odds ratio of being a heavy smoker (95\% confidence intervals) and random variance estimates predicted from access to convenience stores in the 2002-03 New Zealand Health Survey $(\mathrm{n}=12,529)$.

\begin{tabular}{|c|c|c|c|c|}
\hline & $\begin{array}{l}\text { Model } 1^{\mathrm{a}} \\
\text { Baseline }\end{array}$ & $\begin{array}{c}\text { Model } 2^{\mathrm{b}} \\
\text { Individual socio- } \\
\text { economic status }\end{array}$ & $\begin{array}{c}\text { Model } 3^{\mathrm{c}} \\
\text { Area deprivation }\end{array}$ & $\begin{array}{c}\text { Model } 4^{\mathrm{d}} \\
\text { Urban/rural }\end{array}$ \\
\hline \multicolumn{5}{|l|}{ Neighbourhood Access (mins) } \\
\hline Worst $>3.89$ & 1 & 1 & 1 & 1 \\
\hline Worse $1.65-3.89$ & $1.13(0.95-1.35)$ & $1.17(0.98-1.38)$ & $1.10(0.93-1.31)$ & $1.01(0.82-1.24)$ \\
\hline Better 0.98-1.65 & $1.14(0.95-1.37)$ & $1.19(0.99-1.42)$ & $1.07(0.90-1.28)$ & $0.98(0.78-1.21)$ \\
\hline Best $<0.98$ & $1.12(0.94-1.34)$ & $1.15(0.97-1.37)$ & $1.01(0.85-1.20)$ & $0.93(0.75-1.15)$ \\
\hline \multicolumn{5}{|l|}{$\operatorname{Sex}$} \\
\hline Male & 1 & 1 & 1 & 1 \\
\hline Female & $0.79(0.71-0.88)$ & $0.87(0.77-0.98)$ & $0.87(0.77-0.98)$ & $0.87(0.78-0.99)$ \\
\hline \multicolumn{5}{|l|}{ Age group } \\
\hline $65+$ & 1 & 1 & 1 & 1 \\
\hline $45-64$ & $3.56(2.85-4.45)$ & $3.99(3.14-5.09)$ & $3.87(3.04-4.92)$ & $3.89(3.06-4.95)$ \\
\hline $25-44$ & $4.50(3.62-5.59)$ & $5.03(3.91-6.46)$ & $4.87(3.79-6.26)$ & $4.89(3.81-6.28)$ \\
\hline $15-24$ & $2.64(2.02-3.45)$ & $2.62(1.96-3.49)$ & $2.54(1.91-3.39)$ & $2.55(1.91-3.39)$ \\
\hline \multicolumn{5}{|l|}{ Ethnicity } \\
\hline Other & 1 & 1 & 1 & 1 \\
\hline Māori & $1.93(1.67-2.22)$ & $1.59(1.37-1.83)$ & $1.41(1.22-1.63)$ & $1.42(1.23-1.64)$ \\
\hline Pacific & $0.83(0.64-1.07)$ & $0.68(0.52-0.88)$ & $0.57(0.44-0.75)$ & $0.59(0.45-0.76)$ \\
\hline Asian & $0.17(0.11-0.26)$ & $0.17(0.11-0.26)$ & $0.16(0.11-0.25)$ & $0.17(0.11-0.26)$ \\
\hline \multicolumn{5}{|l|}{ Education } \\
\hline Tertiary qualifications & & 1 & 1 & 1 \\
\hline School qualifications only & & $0.98(0.84-1.13)$ & $0.98(0.85-1.13)$ & $0.98(0.85-1.13)$ \\
\hline No qualifications & & $1.46(1.27-1.67)$ & $1.45(1.26-1.66)$ & $1.45(1.26-1.66)$ \\
\hline \multicolumn{5}{|l|}{ Social Class } \\
\hline Managerial/Professional & & 1 & 1 & 1 \\
\hline Other non-manual & & $1.31(1.09-1.58)$ & $1.28(1.06-1.54)$ & $1.28(1.06-1.54)$ \\
\hline Skilled manual & & $2.16(1.77-2.63)$ & $2.08(1.71-2.54)$ & $2.09(1.71-2.54)$ \\
\hline Semi \& unskilled manual & & $1.82(1.49-2.21)$ & $1.73(1.42-2.10)$ & $1.72(1.41-2.09)$ \\
\hline Unavailable & & $1.40(1.13-1.74)$ & $1.33(1.07-1.65)$ & $1.33(1.07-1.65)$ \\
\hline \multicolumn{5}{|l|}{ Benefits } \\
\hline Non-recipient & & 1 & 1 & 1 \\
\hline Recipient & & $1.26(1.10-1.44)$ & $1.24(1.09-1.42)$ & $1.24(1.09-1.41)$ \\
\hline \multicolumn{5}{|l|}{ Household income } \\
\hline$>50,000$ & & 1 & 1 & 1 \\
\hline $25,000-50,000$ & & $1.32(1.14-1.53)$ & $1.22(1.05-1.42)$ & $1.21(1.04-1.41)$ \\
\hline$<25,000$ & & $1.51(1.28-1.79)$ & $1.37(1.16-1.62)$ & $1.37(1.15-1.62)$ \\
\hline \multicolumn{5}{|l|}{ Neighbourhood Deprivation } \\
\hline 1 (least deprived) & & & 1 & 1 \\
\hline 2 & & & $1.23(0.94-1.61)$ & $1.23(0.94-1.61)$ \\
\hline 3 & & & $1.21(0.93-1.58)$ & $1.22(0.94-1.59)$ \\
\hline 4 & & & $1.80(1.41-2.31)$ & $1.80(1.41-2.31)$ \\
\hline 5 (most deprived) & & & $2.17(1.67-2.83)$ & $2.16(1.66-2.81)$ \\
\hline \multicolumn{5}{|c|}{ Neighbourhood Urban/Rural Status } \\
\hline Main urban & & & & 1 \\
\hline
\end{tabular}




\footnotetext{
Secondary urban

$1.20(0.95-1.53)$

Minor urban

$1.10(0.93-1.31)$

Rural centre

$1.04(0.81-1.33)$

Highly rural

$0.90(0.72-1.12)$

Level 2 variance (Std err) $^{\text {e }}$

$0.14(0.04)$

0.09 (0.03)

$0.06(0.03)$

0.04 (0.03)

a. Models include design (to account for sample stratification and oversampling of ethnic minorities), and individual-level sex and age variables.

b. Individual-level socio-economic variables included in models containing design, sex and age variables.

c. Neighbourhood-level deprivation included in models containing individual-level design, sex, age and socio-economic variables.

d. Neighbourhood-level urban area classification included in models containing neighbourhood-level deprivation and individual-level design, sex, age and socio-economic variables.

e. Variance at the neighbourhood-level
}

\section{Discussion}

This paper presents the results of the first national study of the effects of neighbourhood access to outlets selling tobacco on individual smoking behaviour. Two findings are of particular interest. First, we confirmed that, after adjustment for potentially confounding individual-level variables, neighbourhood access to both supermarkets and convenience stores showed a modest association with smoking rates, but this was less evident with respect to the intensity of smoking. Second, although it has previously been noted that tobacco outlets are preferentially located in more deprived New Zealand neighbourhoods[23], after adjustment for neighbourhood variables, residents of neighbourhoods with better geographical access to supermarkets and convenience stores were not more likely to smoke or to be a heavy smoker. Our findings concur with the only similar previous study that also found that after controlling for individual level characteristics high convenience store density remained positively associated with individual smoking but not when neighbourhood SES was taken into account, except in the case of higher income residents.[11]

The main contributions of our study are threefold. Our findings are based on the national and not the local scale, we have captured most outlets selling tobacco, not only convenience stores but also supermarkets and we have separately examined the effects of neighbourhood access on all smokers and heavy smokers. However, our study has a number of limitations. First, there are other sources for procuring tobacco that are not captured in our neighbourhood accessibility measure, including liquor stores and hotels, restaurants, petrol stations that do not sell food, duty free shops, mail order, the internet and other illegal sources. Nonetheless, over 90 percent of smokers in New Zealand acquire their tobacco products from supermarkets, convenience stores or petrol stations.[44] Second, without information on shopping habits there is no validation that people actually purchase tobacco products from their closest supermarket or convenience store. Third, tobacco purchasing may link not only to residential neighbourhood, but also to workplace and other locations. Fourth, the neighbourhood measure of access to tobacco outlets is based on travel times by car but access to a car is itself patterned by neighbourhood socioeconomic characteristics. Finally, the differences in travel time between each quartile are not large, suggesting that few neighbourhoods have substantial distances to travel to an outlet selling tobacco. 
These limitations acknowledged, our results confirm that the effects of access to tobacco outlets on smoking behaviour largely reflect the impact of neighbourhood deprivation. This is not unsurprising given the higher concentration of outlets selling tobacco in disadvantaged neighbourhoods. This association between neighbourhood deprivation and individual smoking is consistent with the findings from a number of countries. Research has persistently found neighbourhood SES or deprivation to be associated with individual smoking, even after individual-level adjustments.[7, 8, 45-47] Previous studies in New Zealand have also found this independent association.[4, 13, 14, 36]

Our findings have a number of important theoretical and policy implications. First, the fact that controls for neighbourhood deprivation effectively removed the relationship between access to tobacco outlets and tobacco consumption confirms the importance of this factor in disadvantaged communities. However, since neighbourhood access is just one contextual feature, more nuanced analyses of the significance of different types of area effects, including neighbourhood incivilities,[17] social capital[48] or local stigma and alienation,[15] are needed in order to more fully understand the key environmental influences which mediate the well known independent relationship between area deprivation and smoking.

Second, although our findings suggest that neighbourhood access to tobacco outlets was not a significant factor in determining individual smoking behaviour, it would be premature to argue against area-based policies restricting the availability of tobacco. First, it remains unclear whether more severe restrictions on the number of tobacco outlets would significantly influence consumption rates, especially if people had to travel longer distances to purchase tobacco. Further analyses involving different travel time thresholds are required. Second, given that many people purchase tobacco from non-neighbourhood outlets (e.g. near workplaces), then the access effects could also be mitigated by restrictions on locating outlets selling cigarettes within important employment nodes (e.g. city centres). Third, in the absence of national-level restrictions on the retail displays of tobacco products, greater local restrictions on tobacco retailing in more deprived areas remain a possibility. Fourth, our cross-sectional analyses have not explored the relationship between access to tobacco products and smoking initiation especially among younger persons in deprived neighbourhoods. Thus further research might usefully examine the relations between smoking stages and sources of cigarettes. Finally, local restrictions on the availability of tobacco could be effective for certain groups, such as solo parents or older people who are more likely to face mobility constraints. Such a possibility may, for example, be one way of reducing the high rates of smoking by Māori women in disadvantaged areas.

Continued research on the pathways linking local area disadvantage to smoking behaviour is warranted. Despite the correspondence between our findings and the earlier (Californian) research, the potential importance of national context should encourage researchers in other countries to examine the influence of neighbourhood resources, such as retail provision, on individual health in their own countries. 
Acknowledgements: We recognise the Crown as the owner, and the New Zealand Ministry of Health as the funder, of the 2002/03 New Zealand Health Survey. We thank Maria Turley and Kylie Mason of Public Health Intelligence, Ministry of Health for preparing the Health Survey data. Finally, we thank the reviewers of the manuscript who provided valuable feedback on our work. 


\section{What this paper adds}

\section{What is already known on this subject?}

- $\quad$ Higher levels of smoking are associated with a range of adverse health outcomes.

- Smoking behaviour is strongly patterned by socioeconomic circumstances with people on lower incomes, lower educational attainment and living in areas of high social deprivation tending to smoke more.

- Neighbourhood access to shops selling tobacco has been suggested as one explanation for the social gradient in tobacco consumption.

\section{What does this study add?}

This paper adds to the understanding of the contextual explanations for smoking behaviour. At the national level, we found little evidence that better geographical access to retailers selling tobacco is associated with higher consumption of cigarettes. The findings of this analysis suggest that area-based restrictions on the availability of outlets selling tobacco alone may not reduce the prevalence of smoking in New Zealand.

\section{Policy Implications}

This study does not find evidence to suggest that restrictions on the neighbourhood availability of tobacco products will be an effective policy instrument in reducing the prevalence of smoking in New Zealand. However, before arguing against area-based policies that restrict the local availability of tobacco, further research is required. In particular, more severe restrictions on the number of tobacco outlets in residential neighbourhoods, and area-based restrictions in non-residential settings (e.g. close to workplaces) may influence the consumption of tobacco. Further, local restrictions on the availability of cigarettes could be effective for certain groups, such as solo parents or older people who are more likely to face mobility constraints. 


\section{References}

1 Office of the Surgeon General. 2004 Surgeon General's Report-The Health Consequences of Smoking. Atlanta, Georgia: U.S. Department of Health and Human Services, Centers for Disease Control and Prevention, National Center for Chronic Disease Prevention and Health Promotion, Office on Smoking and Health 2004.

2 Marmot M. Smoking and inequalities. Lancet 2006;368:341-2.

3 Kawachi I, Berkman LF. Neighborhoods and health. Oxford ; New York: Oxford University Press 2003.

4 Barnett JR. Does place of residence matter? Contextual effects and smoking in Christchurch. N Z Med J 2000;113:433-5.

5 Datta GD, Subramanian SV, Colditz GA, et al. Individual, neighborhood, and state-level predictors of smoking among US Black women: a multilevel analysis. Soc Sci Med 2006;63:1034-44.

6 Diez Roux AV, Merkin SS, Hannan P, et al. Area characteristics, individual-level socioeconomic indicators, and smoking in young adults: the coronary artery disease risk development in young adults study. Am J Epidemiol 2003;157:315-26.

7 Duncan C, Jones K, Moon G. Smoking and deprivation: are there neighbourhood effects? Soc Sci Med 1999;48:497-505.

8 Pearce J, Boyle P, Flowerdew R. Predicting smoking behaviour in census output areas across Scotland. Health Place 2003;9:139-49.

9 Sundquist J, Malmstrom M, Johansson SE. Cardiovascular risk factors and the neighbourhood environment: a multilevel analysis. Int J Epidemiol 1999;28:841-5.

10 Tseng M, Yeatts K, Millikan R, et al. Area-level characteristics and smoking in women. Am J Public Health 2001;91:1847-50.

11 Chuang YC, Cubbin C, Ahn D, et al. Effects of neighbourhood socioeconomic status and convenience store concentration on individual level smoking. J Epidemiol Community Health 2005;59:568-73.

12 Giskes K, van Lenthe FJ, Turrell G, et al. Smokers living in deprived areas are less likely to quit: a longitudinal follow-up. Tob Control 2006;15:485-8.

13 Barnett R, Moon G, Kearns R. Social inequality and ethnic differences in smoking in New Zealand. Soc Sci Med 2004;59:129-43.

14 Barnett R, Pearce J, Moon G. Does social inequality matter? Changing ethnic socioeconomic disparities and Maori smoking in New Zealand, 1981-1996. Soc Sci Med 2005;60:1515-26.

15 Siahpush M, Borland R, Taylor J, et al. The association of smoking with perception of income inequality, relative material well-being, and social capital. Soc Sci Med 2006;63:280112.

16 Bell JF, Zimmerman FJ, Mayer JD, et al. Associations between residential segregation and smoking during pregnancy among urban african-american women. J Urban Health 2007;84:372-88.

17 van Lenthe FJ, Mackenbach JP. Neighbourhood and individual socioeconomic inequalities in smoking: the role of physical neighbourhood stressors. J Epidemiol Community Health 2006;60:699-705.

18 Virtanen M, Kivimaki M, Kouvonen A, et al. Average household income, crime, and smoking behaviour in a local area: the Finnish 10-Town study. Soc Sci Med 2007;64:1904-13. 
19 Fukuda Y, Nakamura K, Takano T. Socioeconomic pattern of smoking in Japan: income inequality and gender and age differences. Ann Epidemiol 2005;15:365-72.

20 Idris BI, Giskes K, Borrell C, et al. Higher smoking prevalence in urban compared to non-urban areas: time trends in six European countries. Health Place 2007;13:702-12.

21 Volzke H, Neuhauser H, Moebus S, et al. Urban-rural disparities in smoking behaviour in Germany. BMC Public Health 2006;6:146.

22 Hyland A, Travers MJ, Cummings KM, et al. Tobacco outlet density and demographics in Erie County, New York. Am J Public Health 2003;93:1075-6.

23 Pearce J, Witten K, Hiscock R, et al. Are socially disadvantaged neighbourhoods deprived of health-related community resources? Int J Epidemiol 2007;36:348-55.

24 Pearce J, Day P, Witten K. Neighbourhood provision of food and alcohol retailing and social deprivation in urban New Zealand. Urban Policy and Research 2008;26:213-27.

25 Scribner R, Cohen D, Kaplan S, et al. Alcohol availability and homicide in New Orleans: conceptual considerations for small area analysis of the effect of alcohol outlet density. J Stud Alcohol 1999;60:310-6.

26 Scribner RA, Cohen DA, Fisher W. Evidence of a structural effect for alcohol outlet density: a multilevel analysis. Alcohol Clin Exp Res 2000;24:188-95.

27 Farrelly M, Bray J. Response to increases in cigarette prices by race/ethnicity, income and age groups - United States - 1976-1993 Morbidity and Mortality Weekly Report 47. Atlanta: Centers for Disease Control and Prevention 1998:605-9.

28 Morland K, Diez Roux AV, Wing S. Supermarkets, other food stores, and obesity: the atherosclerosis risk in communities study. Am J Prev Med 2006;30:333-9.

29 Morland K, Wing S, Diex-Roux A. The contextual effect of the local food environment on residents' diet: the atherosclerosis risk in communities study. Am J Public Health 2002;92:1761-7.

30 Laraia BA, Siega-Riz AM, Kaufman JS, et al. Proximity of supermarkets is positively associated with diet quality index for pregnancy. Prev Med 2004;39:869-75.

31 Pearce J, Hiscock R, Witten K, et al. The contextual effects of neighbourhood access to supermarkets and convenience stores on individual fruit and vegetable consumption. $J$ Epidemiol Community Health 2008;In Press.

32 Cummins S, Petticrew M, Higgins C, et al. Large scale food retailing as an intervention for diet and health: quasi-experimental evaluation of a natural experiment. $J$ Epidemiol Community Health 2005;59:1035-40.

33 Ministry of Health. Tobacco Facts 2003. Wellington: Ministry of Health 2003.

34 Blakely $\mathrm{T}$, Wilson $\mathrm{N}$. The contribution of smoking to inequalities in mortality by education varies over time and by sex: two national cohort studies, 1981-84 and 1996-99. Int J Epidemiol 2005;34:1054-62.

35 Blakely T, Fawcett J, Hunt D, et al. What is the contribution of smoking and socioeconomic position to ethnic inequalities in mortality in New Zealand? Lancet 2006;368:44-52.

36 Moon G, Barnett R. Spatial scale and the geography of tobacco smoking in New Zealand: A multilevel perspective. New Zealand Geographer 2003;59:6-15.

37 Ministry of Health. A Portrait of Health: Key Results of the 2002/03 New Zealand Health Survey. Wellington: Ministry of Health 2004.

38 Pearce J, Dorling D. Increasing geographical inequalities in health in New Zealand, 1980-2001. Int J Epidemiol 2006;35:597-603. 
39 Pearce J, Dorling D, Wheeler B, et al. Geographical inequalities in health in New Zealand, 1980-2001: the gap widens. Aust N Z J Public Health 2006;30:461-6.

40 Ministry of Health. New Zealand Health Strategy. Wellington: Ministry of Health 2000.

41 Pearce J, Witten K, Bartie P. Neighbourhoods and health: a GIS approach to measuring community resource accessibility. J Epidemiol Community Health 2006;60:389-95.

42 Salmond C, Crampton P. NZDep2001 Index of Deprivation. Wellington: Department of Public Health, Wellington School of Medicine and Health Sciences 2002.

43 Department of Statistics. New Zealand Standard Areas Classification Manual 1992. Catalogue No190350092 1992.

44 Ministry of Health. New Zealand Tobacco Use Survey 2006. Wellington: Ministry of Health 2007.

45 Reijneveld SA. The impact of individual and area characteristics on urban socioeconomic differences in health and smoking. Int J Epidemiol 1998;27:33-40.

46 Reijneveld SA. Neighbourhood socioeconomic context and self reported health and smoking: a secondary analysis of data on seven cities. J Epidemiol Community Health 2002;56:935-42.

47 Kleinschmidt I, Hills M, Elliott P. Smoking behaviour can be predicted by neighbourhood deprivation measures. J Epidemiol Community Health 1995;49 Suppl 2:S72-7.

48 Stead M, MacAskill S, MacKintosh A, et al. "It's as if you're locked in": qualitative explanations for area effects on smoking in disadvantaged communities. Health and Place 2001;7:333-43. 